\title{
Fluctuational escape from chaotic attractors in multistable systems
}

\author{
I. A. Khovanov ${ }^{1}$, D. G. Luchinsky ${ }^{1}$, P. V. E. McClintock ${ }^{1}$, \\ and A. N. Silchenko ${ }^{2}$
}

May 30, 2007

\begin{abstract}
${ }^{1}$ Department of Physics, Lancaster University, Lancaster, LA1 4YB, UK.
${ }^{2}$ Institute of Medicine and Virtual Institute of Neuromodulation, Juelich, D-524, Germany.
\end{abstract}

\begin{abstract}
Recent progress towards an understanding of fluctuational escape from chaotic attractors (CAs) is reviewed and discussed in the contexts of both continuous systems and maps. It is shown that, like the simpler case of escape from a regular attractor, a unique most probable escape path (MPEP) is followed from a CA to the boundary of its basin of attraction. This remains true even where the boundary structure is fractal. The importance of the boundary conditions on the attractor is emphasised. It seems that a generic feature of the escape path is that it passes via certain unstable periodic orbits. The problems still remaining to be solved are identified and considered.
\end{abstract}

PACS numbers: 05.45.Gg, 02.50.-r, 05.20.-y, 05.40.-a 


\section{Introduction}

Complex systems are generally noisy and chaotic, both in nature and in a wide range of science and technology. The analysis of stability and the development of methods of control, applicable to chaotic systems in the presence of noise, are therefore of considerable interdisciplinary importance [Bishop \& Xu, 1996; Zhou et al., 2003; Billings et al., 2004]. Examples of applications range from ecology [Sole et al., 1999] to space travel [Serban et al., 2002]. Deep insight into the solution of these problems can be gained from studies of the invariant manifolds that determine the pattern of optimal paths [Graham \& Tél, 1984; Dykman et al., 1996; Luchinsky, 2002] along which a system fluctuates to a given point in configuration space in the limit of large deviations, i.e. where the noise intensity is much smaller then the minimum action required to move the system from the attractor to that point. Particularly important in this context is the role played by the so-called most probable escape path (MPEP) [Dykman \& Krivoglaz, 1979], along which the system escapes from a given basin of attraction. The central idea is finding a wide range of scientific applications including biological ratchets [Luchinsky et al., 2000], dynamical inference of e.g. the cardiovascular system [Smelyanskiy et al., 2005], control of e.g. lasers [Khovanov et al., 2006], and protein folding [Faccioli et al., 2006]. We shall see that the picture applies to chaotic attractors as well as to regular ones, even where the basin structure is fractal.

The conceptual framework is applicable to both flows and maps. It can be developed by following e.g. Grassberger [1989] (see also [Kifer, 1990; Graham et al., 1991]). Consider the $L$-dimensional map

$$
x_{n+1}=f\left(x_{n}\right)+\sigma z_{n}, \quad\left\langle z_{\alpha n} z_{\beta n^{\prime}}\right\rangle=\delta_{n n^{\prime}} \delta_{\alpha \beta},
$$

where $x_{n}, \eta_{n} \in R^{L}$, and $\sigma$ is a row matrix mixing zero-mean white Gaussian sources of noise $z_{n}$. Comparison of (1) with a midpoint approximation for the time evolution of an $L$-dimensional flow on the discrete time $n=1, \ldots, N$

$$
x_{n+1}=x_{n}+h F\left(x_{n}^{*}\right)+\sigma \tilde{z}_{n}, \quad \tilde{z}_{n}=\int_{t_{n}}^{t_{n}+h} \xi(t) d t, \quad\left\langle\tilde{z}_{n} \tilde{z}_{n^{\prime}}\right\rangle=h \hat{I} \delta_{n n^{\prime}}
$$

shows that (2) is a special case of the map (1) with a small parameter $h$, and $x_{n}^{*}=\frac{x_{n}+x_{n+1}}{2}$. Thus the main results obtained below for maps can also be applied to flows, using the following rule: (i) make the substitutions $f\left(x_{n}\right) \rightarrow x_{n}+h F\left(x_{n}^{*}\right)$ and $\sigma \rightarrow \sqrt{h} \tilde{\sigma}$; and (ii) take the limit $h \rightarrow 0$. In the particular case of large deviations, we are interested in the minimum of the energy functional under the condition $x_{n+1}-f\left(x_{n}\right)-\sigma z_{n}=$ 0 . It is intuitively clear that $S$ is a non-decreasing function along the trajectory and must be at least piece-wise differentiable in the limit $h \rightarrow 0$, thus justifying its use as a generalized potential for non-equilibrium systems [Graham \& Tél, 1984; Jauslin, 1987]. By introducing Lagrange multipliers $\lambda_{n}$ and varying the effective functional

$$
S^{*}=\frac{1}{2} \sum_{n=1}^{N} \xi_{n}^{T} \xi_{n}+\sum_{n=1}^{N} \lambda_{n}^{T}\left(x_{n+1}-f\left(x_{n}\right)-\sigma \xi_{n}\right)
$$

with respect to $\xi_{n}, \lambda_{n}$, and $x_{n}$ (cf. [Grassberger, 1989; Beri et al., 2005]) one obtains the equations of motion of the system along optimal paths in the course of large deviations 
in the form of an area-preserving map

$$
x_{n+1}=f\left(x_{n}\right)+\sigma \sigma^{T} \lambda_{n}, \quad \lambda_{n+1}=\left[f^{\prime}\left(x_{n+1}\right)\right]^{-1 T} \lambda_{n} .
$$

Translating this result for a flow system [Beri et al., 2005] we obtain

$$
\dot{x}=F(x)+D^{\prime} p, \quad \dot{p}=-F^{\prime}(x) p,
$$

where $D=\tilde{\sigma} \tilde{\sigma}^{T}$, and in the $h \rightarrow 0$ limit we have introduced the notations $\lambda_{n} \rightarrow p(t)$, $x_{n} \rightarrow x(t),\left(\lambda_{n+1}-\lambda_{n}\right) / h \rightarrow \dot{p}$, and $\left(x_{n+1}-x_{n}\right) / h \rightarrow \dot{x}$. Note that the Hamiltonian equations thus obtained (with Hamiltonian $H=\frac{1}{2} p^{T} D p+p^{T} F$ ) for the optimal paths are known from a WKB-like approximation of the Fokker-Planck equation [Cohen \& Lewis, 1967; Ventcel \& Freidlin, 1970].

The application of (4) and (5) to analysis of the stability and control of chaotic flows and maps requires knowledge of the boundary conditions. In particular, the stability of an attractor is determined by the probability of escape from its basin of attraction along the MPEP. Similarly, restriction of the system dynamics within the basin of a given attractor is enforced by a control function that steers the system to its boundary. In the particular case of energy optimal control, the problems of stability and control can both be reduced to boundary value problems for auxiliary Hamiltonian flows (5) [Luchinsky et al., 1998] or area preserving maps (4) [Khovanov et al., 2000]. It is clear therefore that analysis of the boundary conditions is crucial for the solution of both problems. Formally, the boundary conditions are set at the attractor (for $t \rightarrow-\infty$ ) and at the boundary (for $t \rightarrow+\infty$ ). At the end points both $\lambda$ and $p$ are zero.

In the case of chaotic dynamics, however, the geometrical structure of the attractor (and also the boundary in cases where this is fractal) is very complex. And it is not known a priori if the boundary conditions in chaotic systems can be specified uniquely or, indeed, whether a unique MPEP exists at all. Consequently, the fluctuational escape problem in chaotic systems has attracted a great deal of attention during the last two decades (see e.g. [Beale, 1989; Grassberger, 1989; Kifer, 1990; Graham et al., 1991; Reimann et al., 1994; Reimann \& Talkner, 1995; Kraut \& Feudel, 2003]). Here we present a brief review of recent progress in understanding the boundary conditions for escape from a CA, both with and without fractal boundaries [Luchinsky \& Khovanov, 1999; Khovanov et al., 2000; Luchinsky, 2002; Silchenko et al., 2003a; Khovanov et al., 2003; Kraut \& Grebogi, 2004; Beri et al., 2005; Silchenko et al., 2005] It turns out that the boundary conditions at the attractor and on its fractal boundary can be identified uniquely with certain unstable periodic orbits. This in turn opens up the possibility of developing a general approach to the solution of the stability and control problems by application of a WKB-like approximation. To identify boundary conditions on the attractor a method based on the prehistory probability distribution Dykman et al. [1992] can conveniently be employed [Dykman et al., 1996; Luchinsky et al., 1997; Luchinsky, 2002; Silchenko et al., 2005]. The exact locations of the unstable periodic orbits can be found by use of the standard methods of nonlinear dynamics [Schmelcher \& Diakonos, 1997]. It has also been found recently that in some quite general situations the boundary conditions can be identified directly from the known structure of non-hyperbolic attractors and fractal boundaries [Silchenko et al., 2003a; Kraut \& Grebogi, 2004].

Once the boundary conditions are identified, the MPEP can be found as the path that both minimizes the energy functional (3) and also belongs to the unstable manifold of 
the boundary orbit embedded within the attractor $\mathcal{M}_{A}^{U}$ and the stable manifold $\mathcal{M}_{B}^{S}$ of the boundary orbit on the attractor boundary. Analyses of the structure of $\mathcal{M}_{A}^{U}$ and $\mathcal{M}_{B}^{S}$ can to some extent be carried out in parallel [Maier \& Stein, 1997]. As an example [Beri et al., 2005], consider the parameterizations of $\mathcal{M}_{A}^{U}$ in the close vicinity of the stable point $S$ of period one, where the map (4) can be linearized as

$$
\left[\begin{array}{c}
\delta_{n+1} \\
\lambda_{n+1}
\end{array}\right]=M\left[\begin{array}{c}
\delta_{n} \\
\lambda_{n}
\end{array}\right], \quad M=\left[\begin{array}{cc}
f^{\prime}(S) & I \\
0 & {\left[f^{\prime}(S)\right]^{-1 T}}
\end{array}\right]
$$

where $\delta_{n}$ is a small deviation from $S$ and the corresponding "momentum" $\lambda_{n} \sim O\left(\delta_{n}\right)$. Because of its area-preserving property (note the structure of $M$ in Eq. (6)), $M$ has the same number $L$ for contracting $\alpha_{s}$ and expanding $\alpha_{u}$ eigenvalues. Clearly, $\mathcal{M}_{A}^{U}$ is spanned by the eigenvectors corresponding to the expanding eigenvalues. Then an arbitrary point on $\mathcal{M}_{A}^{U}$ can be written as

$$
\left[\begin{array}{c}
\delta_{n+1} \\
\lambda_{n+1}
\end{array}\right]=\sum_{l=1}^{L} c_{l} \alpha_{u, l}^{n}\left[\begin{array}{c}
e_{u, l}^{\delta} \\
e_{u, l}^{\lambda}
\end{array}\right]
$$

where $e_{u, l}^{\delta}$ and $e_{u, l}^{\lambda}$ are the $L$-dimensional coordinate and "momentum" components of the $2 L$-dimensional eigenvector corresponding to the expanding eigenvalue $\alpha_{u, l}$. It can be seen from (7) that $\mathcal{M}_{A}^{U}$ of the $2 L$-dimensional map (4) can be parameterized with $L$ parameters $c_{l}$. For a small deviation $\delta$ chosen in an $L$-dimensional spherical shell around $S$, the corresponding effective momenta can be found as

$$
\lambda_{n}=\left[e_{u, l}^{\lambda}\right]\left[e_{u, l}^{\delta}\right]^{-1} \delta_{n},
$$

where $\left[e_{u, l}^{\lambda}\right]$ and $\left[e_{u, l}^{\delta}\right]^{-1}$ are $L \times L$ matrices composed of $L$-dimensional column vectors $e_{u, l}^{\lambda}$ and $e_{u, l}^{\delta}$.

Similar results can be obtained for the flow (2). One must bear in mind that, in the continuous case, $\mathcal{M}_{A}^{U}$ can be characterized by $(L-1)$ parameters because, for the system (5), one of the eigenvalues corresponding to motion along the limit cycle is necessarily 0 . In practice, to parameterize $\mathcal{M}_{A}^{U}$ in the continuous, case one has: (i) to identify the boundary limit cycle; and (ii) to introduce a Poincaré cross-section for this limit cycle and a corresponding map. The parameterizations of $\mathcal{M}_{A}^{U}$ in the Poincaré cross-section can then be performed following Eqs. (6)-(8).

Below we focus exclusively on analysis of the boundary conditions. The closely related problem of the analysis of invariant measures on chaotic attractors is not considered here (see, however, [Jung \& Hänggi, 1990; Reimann et al., 1994; Reimann \& Talkner, 1995; Beri et al., 2005]).

We will describe and discuss recent progress in the analysis of the escape problem in chaotic systems, treating escape over regular and fractal basin boundaries in Secs. 2 and 3 respectively. We summarise the status of the subject and identify the main problems still to be solved in Sec. 4.

\section{Chaotic escape over a regular basin boundary}

We now consider and compare noise-induced escape from the basins of quasi-hyperbolic and non-hyperbolic CAs. The property of hyperbolicity means that, at each point of 
a chaotic set, the tangent space can be split into expanding and contracting subspaces (manifolds), and that the angle between the subspaces differs from zero. In case of a quasi-hyperbolic attractor, the property hyperbolicity is broken into a finite number of points. In a non-hyperbolic attractor, homoclinic tangencies between stable and unstable manifolds take place at an infinite numbers of points. The homoclinic tangencies form a homoclinic structure which is structurally unstable [Smale, 1966] and can be changed completely by a small variation of parameters [Gonchenko et al., 1997]. CAs are typically non-hyperbolic.

We suppose the basins of attraction to be smooth and that one can distinguish a boundary set. Within the framework of large fluctuation theory [Freidlin \& Wentzel, 1984] we expect escape via the boundary set in the zero-noise limit. Another boundary has to be chosen on the CA. In the case of a quasi-hyperbolic attractor we have a density of saddle cycles; the trajectory is bounded by manifolds of the cycles and there is a very small (in comparison with the whole attractor) region that is non-hyperbolic. For a nonhyperbolic attractor we cannot specify the sets composing the attractor. Moreover it has been shown [Afraimovich et al., 1983] that a typical non-hyperbolic attractor can be considered as a quasi-attractor composed of several sets including hyperbolic and stable ones; complex behaviour is induced by round-off errors in computer simulations or by noise in experiments. In the neither case can we specify a priori any particular set as the boundary condition. In the case of a fractal boundary (see Sec. 3) there is an immediate question: which boundary set is the boundary for the optimal escape path? In addition, noise can significantly change the probability distribution on the attractor [Anishchenko et al., 2001; Schroer et al., 1998]. It is obvious that in such a situation a direct solution of the equation (5) is problematic. We have therefore used an experimental approach to solve the boundary problem.

The approach proposed by Dykman et al. [1992] is based on the fact that the optimal path is not a theoretical abstraction: on the contrary, the path corresponds to a real trajectory that can be observed in an experiment. The approach consists in making measurements of the prehistory probability distribution $p_{h}\left(\mathbf{x}, t ; \mathbf{x}_{f}, t_{f}\right)$ in which information is gathered on all trajectories in a close neighborhood of the chosen state $\mathbf{x}_{f}$ outside the attractor. Luchinsky [1997] has demonstrated that the approach allows one to determine the optimal fluctuational force as well. For our task the prehistory approach allows us to avoid the question of boundary conditions mentioned above, and of the distribution near the CA, and simultaneously determines the boundary condition on the CA.

As an example of a multistable system with a non-hyperbolic attractor, we chose a non-autonomous nonlinear oscillator and, as a system with a quasi-hyperbolic attractor, we selected the Lorenz equations with white noise included additively in one variable.

We first consider escape from the quasi-hyperbolic attractor in the Lorenz system. The latter was originally obtained as a simplified model to describe convection of a fluid between two planes at different temperatures, where the thermal and gravitational gradients are oppositely directed [Lorenz, 1963]. Later it was considered as a model for the single mode laser [Haken, 1975; Weiss et al., 1988, 1995]. It is described by the following equations:

$$
\dot{x}_{1}=\sigma\left(x_{2}-x_{1}\right), \dot{x}_{2}=r x_{1}-x_{2}-x_{1} x_{3}, \dot{x}_{3}=x_{1} x_{2}-b x_{3}+\sqrt{D} \xi(t),
$$

Here, $x_{1}, x_{2}$ and $x_{3}$ are the dynamical variables; and $\sigma, r$ and $b$ are parameters. For simplicity we add a white-noise source $\xi(t)$ of intensity $D$ only to the third equation 

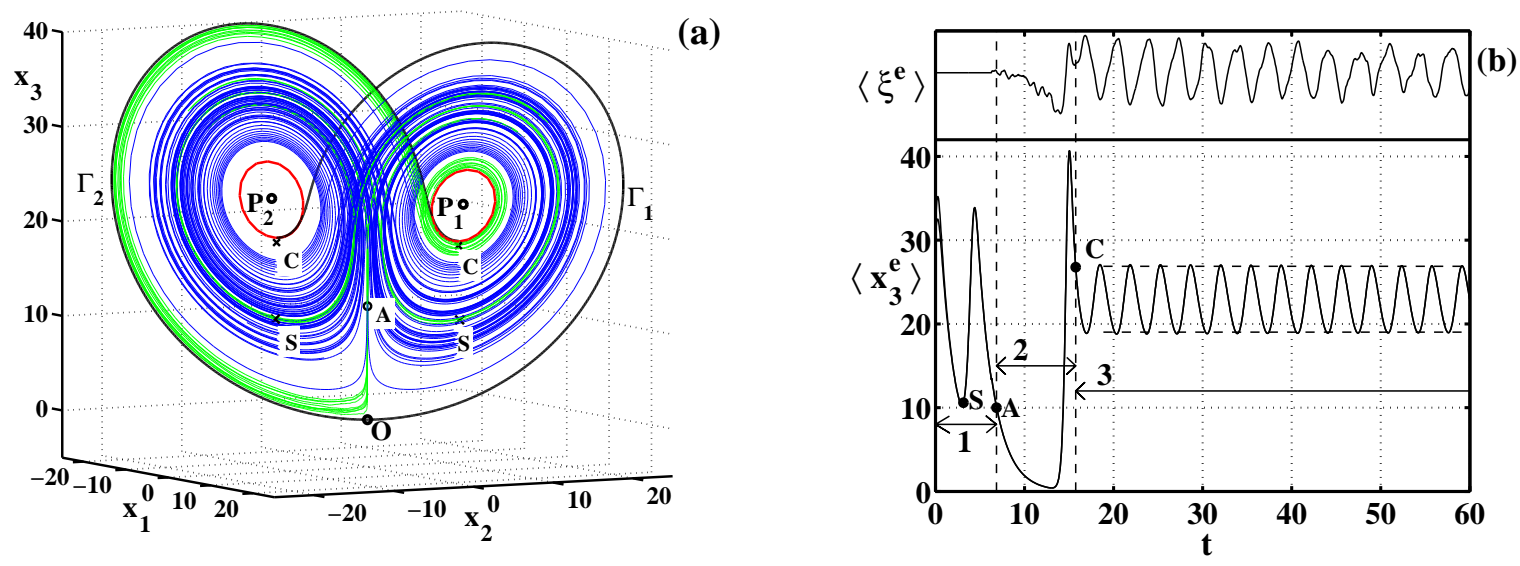

Figure 1: (a) The structure of phase space of the Lorenz system. The stable points $P_{1}$ and $P_{2}$, and the saddle point $\mathrm{O}$ are shown by filled circles. The saddle cycles $L_{1}$ and $L_{2}$ are shown by the red line, the separatrices $\Gamma_{1}$ and $\Gamma_{2}$ are shown by black lines. The blue line corresponds to a trajectory of the quasihyperbolic attractor. Several typical escape trajectories are shown by green lines. (b) The coordinate of the averaged escape trajectory $\left\langle x_{3}^{e}\right\rangle$ (bottom) and the averaged fluctuational force $\left\langle\xi^{e}\right\rangle$ (top), corresponded to the optimal path and the optimal force, respectively. The three stages of escape are indicated.

of system (9); this does not violate the mirror symmetry of the system. We set the parameters as $\sigma=10, b=8 / 3, r=24.08$, so that two stable equilibrium points $P_{1}$ and $P_{2}$ coexist with a CA as shown in Fig. 1(a). The saddle cycles, with stable manifolds transverse to the attractor, are dense everywhere in the Lorenz CA. Apart from the saddle cycles, the chaotic set also contains the separatrices $\Gamma_{1}$ and $\Gamma_{2}$ and the saddle point $O$. The existence of a saddle point in the CA defines the non-hyperbolicity property and the prefix "quasi" in the definition of a quasi-hyperbolic CA [Afraimovich et al., 1983].

The boundaries of the CA are specified by the initial segments of the separatrices $\Gamma_{1}$ and $\Gamma_{2}$ that closely approach the saddle cycles $L_{1}$ and $L_{2}$ (see Fig. 1(a)), and which theoretically [Afraimovich et al., 1983] belong to the attractor. However, numerical studies have shown that the probability of the noise-free system passing near the separatrices $\Gamma_{1}$ and $\Gamma_{2}$ or the cycles $L_{1}$ and $L_{2}$ is exponentially small. In fact, in the noise-free system, no trajectories could be found approaching a close vicinity of these cycles along the separatrices on any reasonable timescale of observation (a few weeks in our case).

Very weak noise $(D=0.001)$ can induce transitions from the CA to the states $P_{1}$ or $P_{2}$, so that the Lorenz attractor becomes metastable. We build prehistory distributions both for the escape trajectories and for the corresponding realizations of the random force. For definiteness, we examine escape to the stable point $P_{1}$.

Analysis of the prehistory distribution of the escape trajectories by Anishchenko et al. [2002] shows that there is well-defined maximum in their distribution. We can therefore define the optimal path by ensemble-averaging the escape trajectories. The optimal fluctuational force can be obtained by averaging the corresponding realizations of the random force. Fig. 1(b) shows the coordinate $x_{3}$ of the optimal path (MPEP), and the value $p_{3}$ of the averaged fluctuational force, as functions of time. The results obtained suggest the following scenario of escape from the Lorenz attractor. The escape path consists of the three main parts shown in Fig. 1(b): (i) the deterministic part from point $S$ to point $A$; (ii) a part corresponding to fast fluctuation-assisted motion along the separatrices from point $A$ to point $C$; and (iii) slow diffusion from point $C$ to overcome the deterministic 

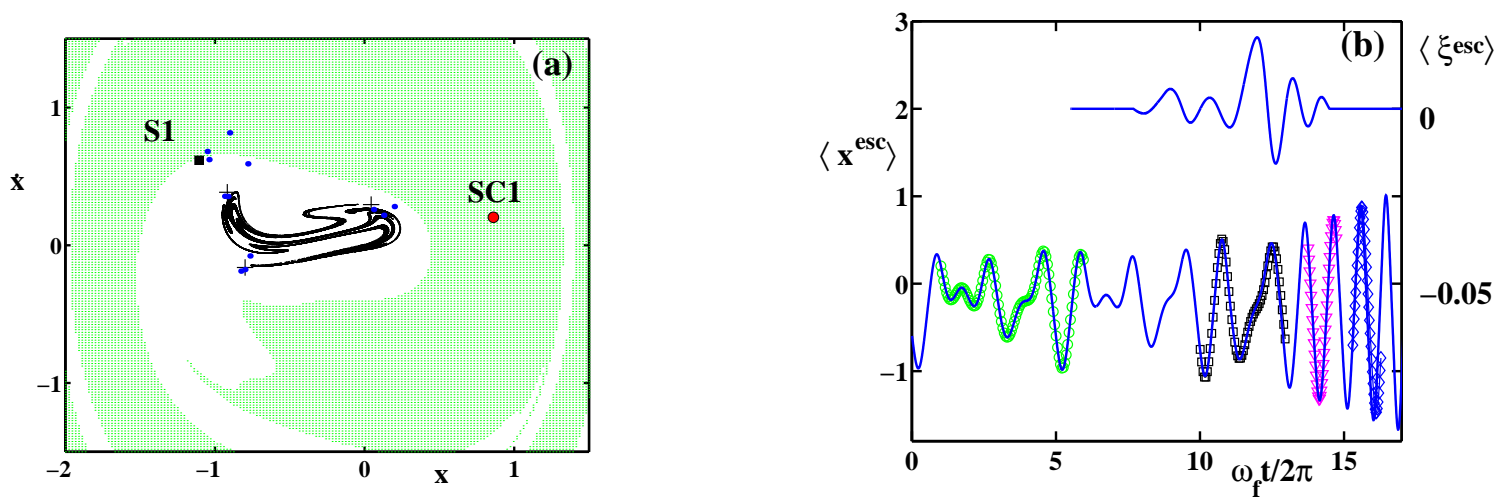

Figure 2: (a) The structure of phase space of (10) in Poincaré section. The basins of attraction of the stable cycle $S C_{1}$ (red filled circle) and the chaotic attractor (small black dots) are shown in green and white respectively. The saddle cycle $S_{1}$ is shown by the black filled square, and the saddle cycle $S_{3}$ is shown by the black pluses. Intersections of the actual escape trajectory with the Poincaré cross-section are indicated by blue circles. (b) The coordinate of the averaged escape trajectory $\left\langle x_{1}^{e s c}\right\rangle$ (bottom) and the averaged fluctuational force $\left\langle\xi^{e s c}\right\rangle$ (top), corresponding to the optimal path and the optimal force. Single periods of the saddle cycles $S_{5}, S_{3}$ and $S_{1}$ are indicated by green circles, black squares and magenta triangles respectively; the stable cycle $S C_{1}$ is shown by blue rhombs.

drift on the unstable manifold of the saddle cycle $L_{1}$ and to cross $L_{1}$. It is important to note that noise plays a different role during each part of the escape [Anishchenko et al., 2002].

Thus, for a quasi-hyperbolic attractor, there exists a unique optimal escape path. The role of fluctuations is two-fold. First, they deliver the system to a seldom-visited region in the neighborhood of the saddle cycle $L_{1}$. Secondly they induce the system to cross the saddle cycle $L_{1}$. The analysis does not indicate any specific sets that can be viewed as the boundary condition on the CA, but it clearly shows that the escape process is closely connected with the non-hyperbolic structure of attractor: the stable and unstable manifolds of the saddle point $O$.

We now discuss escape from a non-hyperbolic attractor in the periodically driven nonlinear oscillator described by:

$$
\ddot{x}=-2 \Gamma \dot{x}-\omega_{0}^{2} x-x^{2}-x^{3}+h \sin \left(\omega_{f} t\right)+\sqrt{D} \xi(t) .
$$

Here $x$ corresponds to the oscillator coordinate; $\Gamma$ and $\omega_{0}$ are oscillator parameters; $h$ and $\omega_{f}$ are the amplitude and frequency of external driving. This form of nonlinear oscillator is encountered in many applications [Soskin et al., 1997]. We fixed the parameters $(\Gamma=$ 0.025, $\left.\omega_{0}=0.597, h=0.13, \omega_{f}=0.95\right)$ in the region where the CA and a stable limit cycle $S C_{1}$ coexist as shown in Fig. 2(a). The CA appears as the result of period-doubling bifurcations, and thus corresponds to non-hyperbolic attractor [Gonchenko et al., 1997]. Unfortunately, in contrast to the Lorenz system, there is no clear description of the structure of the non-hyperbolic attractor.

The boundary between the attractors is formed by stable manifolds of the saddle cycle of period $1\left(S_{1}\right)$. The filled circles in Fig. 2(a) show the intersections of one of the real escape trajectories with the given Poincaré section. Analysis of the prehistory distribution [Luchinsky \& Khovanov, 1999; Luchinsky, 2002] showed that most probable escape path starts at the saddle cycle of period $5\left(S_{5}\right)$ embedded in the attractor, passes through the saddle cycle of period $3\left(S_{3}\right)$ and finishes on the saddle cycle $S_{1}$ at the boundary of 
the basin of attraction. It can be seen in Fig. 2(b) that the optimal force switches on at the moment when the system leaves $S_{5}$ along its unstable manifold and switches off again when the system reaches the boundary cycle $S_{1}$. Finite action is required to move system from $S_{5}$ to $S_{3}$, and additional analysis showed that the cycle $S_{3}$ does not belong to the CA, although it lies close to the attractor boundary. Additionally we found that for a very small noise intensity (insufficient to observe transitions to $S_{1}$ on a reasonable time scale) a large fluctuation from the CA corresponds to a transition from the cycle $S_{5}$ to the cycle $S_{3}$ and, moreover, trajectories reaching $S_{3}$ have all passed through $S_{5}$. The probability of finding the system on $S_{5}$ does not lie within the highest probability region of the attractor, but neither is $S_{5}$ in the exponential tail of the distribution in the presence of noise. With increasing noise intensity, the probability of finding the system in the vicinity of $S_{5}$ increases. It is important to note that, although $S_{5}$ is in the low-probability region of the attractor, the transient time $t_{r}$ required for the system to reach $S_{5}$ (in the presence of noise) from an arbitrary initial condition on the attractor is still exponentially smaller than the average escape time $t_{e}$.

Thus, the experimental approach based on an analysis of the prehistory of large fluctuations allows us: (i) to conclude that escape from a non-hyperbolic attractor occurs in a sequence of jumps between saddle cycles; (ii) to specify the boundary condition on the $\mathrm{CA}$; and (iii) to determine the energy-minimal (optimal) fluctuational force controlling the transition between attractors. Note that we confirmed the results of the prehistory approach by numerical solution of (5) with the boundary conditions set on the cycles $S_{5}$ and $S_{1}$ [Luchinsky, 2002].

It is instructive to compare the escape processes from quasi-hyperbolic and nonhyperbolic attractors. We stress two points.

(i) To determine the optimal path corresponding to the solution of (5) we applied the prehistory approach. It allows us to determine the trajectory corresponding to a global minimum of the escape energy if the relaxation time to an equilibrium distribution on the attractor, $t_{r}$, is much shorter than the fluctuational escape time from the attractor, $t_{e s c}: t_{r} \ll t_{e s c}$. This escape time increases exponentially with decreasing noise intensity $D$ as $t_{e s c} \propto \exp (S / D)$, where $S$ is the activation "energy". Since $D$ is always finite in practice (because observation times are necessarily finite), the satisfaction of the condition $t_{r} \ll t_{e s c}$ is primarily related to $t_{r}$, i.e. to the properties of the equilibrium distribution on the attractor. For the non-hyperbolic CA in the periodically-driven nonlinear oscillator, the noise intensity can be chosen in such a way that the condition $t_{r} \ll t_{e s c}$ is satisfied. In this case, the optimal trajectory found does not depend on the initial conditions on the attractor or on the noise intensity. For the Lorenz system, the situation is different. As pointed out above, the probability of the system being in the neighborhood of the manifolds of the saddle point $O$ is exponentially small. It may be said that the equilibrium distribution function on the attractor has tails similar to those of a Gaussian distribution, and that the trajectory only rarely falls within these tails. Moreover, our reasoning that the trajectory must come arbitrarily close to the saddle-point manifolds when $t \rightarrow \infty$ is based on a theoretical analysis of the Lorenz system [Afraimovich et al., 1983], whereas numerical studies show that this probability is effectively zero for sufficiently long but finite computation times. This implies that the relaxation time to an equilibrium distribution on a quasi-hyperbolic CA is much longer than any realistically feasible observation time. Consequently, for the Lorenz system, we have 
to investigate the dynamics of fluctuational escape within a finite time interval: the question of how the solution obtained depends on the initial conditions on the attractor, and on the noise intensity, still remains an open question (cf. the studies of nonequilibrium trajectories [Soskin, 1999]). However, the experimentally found escape scenario (see below) suggests that a decrease in noise intensity cannot result in qualitative changes of the escape trajectory. Thus, by using the prehistory approach for the quasi-hyperbolic attractor we effectively reformulate the boundary task with infinite time to the corresponding task with finite time. This reformulation results from the structure of the quasi-hyperbolic attractor and, on the basis of the results obtained we conclude that, for multi-stability in the Lorenz system, the boundary of the CA and those of the stable cycles coincide, i.e. the stable manifolds of the boundary cycles $L_{1}$ and $L_{2}$ are formed by manifolds of the saddle point $O$. Theoretically, therefore, zero action is required to bring the system into the boundary set whereas, in practice, we need additional non-zero action to keep and move the system along the manifolds in the non-hyperbolic region of the CA. This latter feature means that noise significantly changes the tails of a quasi-hyperbolic attractor in the vicinity of a non-hyperbolic region.

(ii) In the case of a non-hyperbolic attractor, its non-hyperbolicity plays an essential role in the escape process. For a non-hyperbolic attractor, saddle cycles embedded in the attractor and basin of attraction are important. We found that the cycles involved in the escape have low period numbers and that the cycle corresponding to the boundary condition on the attractor has a low residence probability in the absence of noise. These observations allow us to claim that these cycles are connected with the non-hyperbolic structure of the CA or with homoclinic tangencies (bifurcations). The last conclusion is supported by observations first, that noise-induced deformation occurs in the vicinity of homoclinic tangencies [Jaeger \& Kantz, 1997] and, secondly, that the escape path from the non-hyperbolic attractor in the Henon map lies in a small vicinity of the primary homoclinic tangency closest to the basin boundary [Kraut \& Grebogi, 2004]; note, however, that the latter authors go beyond the rigorous Hamiltonian theory by ignoring the questions about boundary conditions on the CA. Thus, for both quasi-hyperbolic and non-hyperbolic attractors, escape trajectories are intimately connected with the non-hyperbolic structure of the attractors.

\section{Chaotic escape over a fractal basin boundary}

The above results demonstrate that, in the case of a smooth basin boundary, a strong relationship exists between the structure of a chaotic attractor and the structure of the optimal escape path. The problem of finding the boundary conditions would appear to become far more difficult where two co-existing CAs are separated by a fractal basin boundary (FBB). In this case, the complex fractal structure of the basin boundary is determined by a chaotic saddle: it results from homoclinic intersections of the stable and unstable manifolds of the regular saddle point, originally forming a smooth basin boundary.

We now consider the mechanism of fluctuational escape across an FBB. To model the process, we examine the two-dimensional Holmes map driven by the zero-mean white 

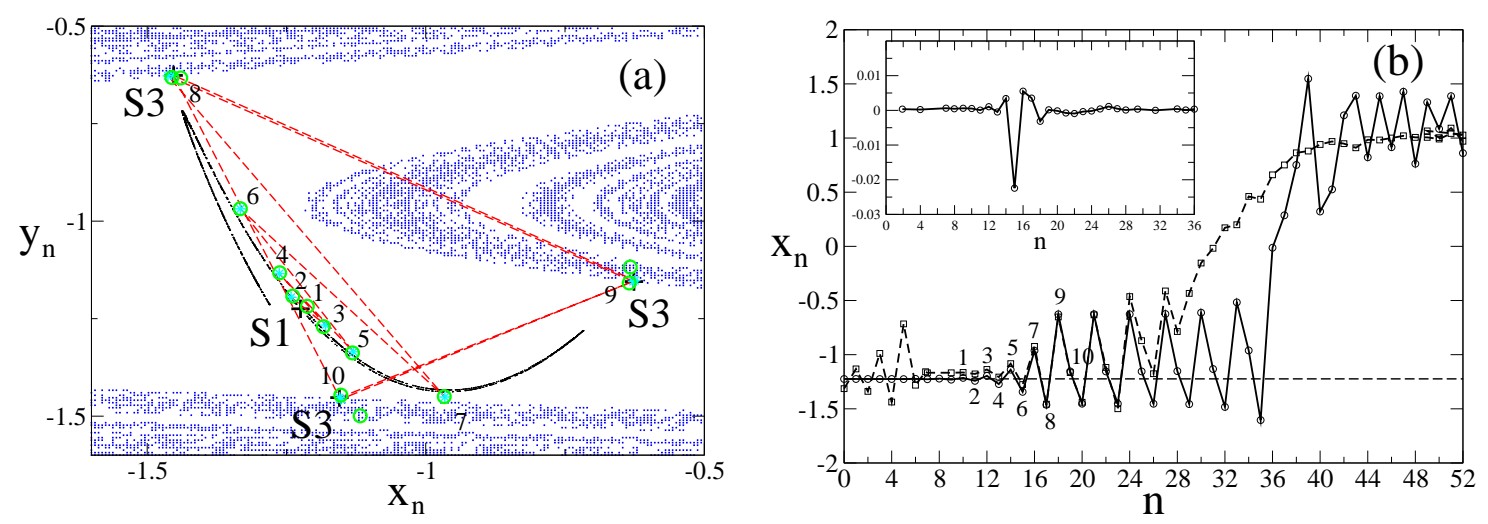

Figure 3: (a) The structure of phase space of (11) in Poincaré section. One of the two co-existing CAs is indicated by the centrally-placed filled curve. Its basin of attraction is shown in white, whereas points belonging to the other basin are shaded in blue. The most probable escape path connecting the CA with the period-3 saddle cycle $S 3$ lying on the fractal boundary is shown by the sequentially numbered small green circles (from solution of the boundary value problem) and stars (from Monte-Carlo simulations) connected by the red dashed line. (b) The $x$-coordinates of the optimal escape paths shown in (a) which were obtained both from the Monte-Carlo simulations with $D=10^{-5}$ (dashed line) and from the solution of the boundary value problem (solid line). The abscissa of the saddle point $S 1$ is shown by the thin-dashed line. The inset shows the optimal fluctuational force moving the system (11) to the FBB.

Gaussian noise $\xi_{n}$ of variance $D$ :

$$
\begin{aligned}
& x_{n+1}=y_{n} \\
& y_{n+1}=-b x_{n}+d y_{n}-y_{n}^{3}+\xi_{n} .
\end{aligned}
$$

When noise-free, the system (11) has pairs of co-existing attractors the basins of which are separated by a boundary that may be either smooth or fractal, depending on the chosen values of parameters. The properties of (11), including the structures both of its $\mathrm{CA}$ and of its locally disconnected FBB, are generic for a wide class of maps and flow systems [McDonald et al., 1985]. We chose for our studies $b=0.2$ and $d=2.7$, which corresponds to there being two co-existing CAs with basins separated by an FBB. Each CA consists of two disconnected parts and appears as the result of a period-doubling cascade. One of them is shown in Fig. 3(a).

To find the optimal escape path, we follow the above approach, exciting a numerical model of the system (11) with weak noise and collecting both the escape trajectories between the CAs and the corresponding realizations of noise that induced them. By averaging a few hundred such trajectories and noise realizations, we were able to determine both the optimal escape path and the corresponding optimal force, which are presented in Fig. 3(b). It can be seen that the system (11) leaves the CA, falling into a small neighborhood of the saddle point of period $1(S 1)$ located between its two disconnected parts. Its stable manifolds separate the parts of the CA, while the unstable ones approach the CA. The system then makes a few iterations in some small neighborhood of $S 1$ (the small plateau in Fig. 3(b)) and then moves in three steps to the FBB, crossing it at the saddle point of period $3(S 3)$. Calculations have shown that, for the chosen parameter values, $S 3$ lies on the FBB. Moreover, its stable manifold (solid black line in Fig. 3) is dense in the FBB and detaches the open neighborhood including an attractor from the FBB itself, thus allowing us to classify it as an accessible boundary point [Grebogi et al., 1987; Silchenko et al., 2003b,a]. It should be noted that the boundary point $S 3$ is the only 
saddle point belonging to the homoclinic structure whose stable and unstable manifolds are not tangent to each other. All other homoclinic points are buried in the FBB and inaccessible from the open neighborhood including the $\mathrm{CA}$, because of such tangencies. Moreover, in the absence of noise, $S 3$ is the only saddle point on the FBB from which the noise-free system (11) can relax to an attractor in a finite number of iterations. For all other initial conditions, taken at inaccessible saddle points, the noise-free system (11) must stay inside the FBB for an infinitely long interval [Grebogi et al., 1987]. In other words, the saddle point $S 3$ is closest to the CA's homoclinic point whose stable manifold separates the interior (including the $\mathrm{CA}$ ) from the FBB. Thus, the results of this statistical analysis allow us to determine both the boundary conditions near the CA and the FBB, and to demonstrate the uniqueness of the optimal escape path [Silchenko et al., 2003a, 2005].

Furthermore, an analysis of the structure of escape trajectories inside the FBB allows us to clarify the role of the homoclinic saddle points resulted from an infinite sequence of saddle-node bifurcations of period 3,4,5,6,7.., at parameter values $d_{3}<d_{4}<d_{5}<d_{6}<$ $d_{7} \ldots$ [Grebogi et al., 1987]. These homoclinic orbits were classified as so-called original saddles, and it was shown that their stable and unstable manifolds cross each other in a hierarchical sequence [Grebogi et al., 1987]. This deterministic structure of the manifolds of the original saddles is robust to noise and determines the escape paths inside the FBB. Indeed, to escape from a CA, the system must first cross the stable manifold of the accessible orbit, and then the stable manifolds of the other original saddles, in a predetermined hierarchical sequence. Once the system crosses the stable manifold of a saddle orbit it relaxes noise-free to the corresponding orbit, which it then leaves along its unstable manifold. We have shown that the hierarchical interrelation between original saddles involved in the escape is closely linked to the eigenvalues of the Jacobian at these saddles, characterizing their local stability with respect to motion on the manifolds [Silchenko et al., 2003a, 2005].

To find the MPEP connecting two co-existing CAs, we used the Hamiltonian formalism described in Sec. 1. Minimization is performed over all the possible realizations of noise $\left\{\xi_{n}\right\}$ that induce a transition of the system (11) from the CA (with the initial condition on $S 1$ ) to the FBB (with the final condition on the accessible orbit $S 3$ ). It is equivalent to minimization of the auxiliary cost function (3) which results in the following areapreserving map:

$$
\begin{aligned}
x_{n+1} & =y_{n} \\
y_{n+1} & =-b x_{n}+d y_{n}-y_{n}^{3}+\lambda_{n}^{y} \\
\lambda_{n+1}^{x} & =\left(d-3 x_{n+1}^{2}\right) \lambda_{n}^{x} / b-\lambda_{n}^{y} / b \\
\lambda_{n+1}^{y} & =\lambda_{n}^{x}
\end{aligned}
$$

The numerical results obtained for the system (12) are presented in Fig. 3(b). It can be seen that the MPEP calculated via minimization of the energy functional practically coincides with the optimal escape path obtained from Monte-Carlo simulations.

That the above results are generic has been confirmed by numerous studies of fluctuational transitions across FBBs in different dynamical systems. In particular, the described scenario of fluctuational escape was observed in all multistable systems whose basins of attraction in phase space are separated by locally-disconnected FBBs, which is the most 
frequently observed type of FBB [Silchenko et al., 2003a, 2005; Khovanov et al., 2006]. Moreover, studies of fluctuational transitions in a dynamical system exhibiting a "fractalfractal" boundary crisis showed that the accessible boundary point can effectively be moved inside the domain of attraction [Silchenko et al., 2005]. It has therefore become possible to predict a scenario of escape through a fractal boundary using a deterministic analysis of the FBB structure. Furthermore, recent results [Kraut \& Grebogi, 2004] have shown that the boundary conditions at CAs can also be related to the homoclinic structure, and thus found deterministically.

\section{Conclusion}

We have described results which allow us to conclude that, for a large class of chaotic flows and maps, there exists a unique most probable path along which the system escapes from the basin of attraction of a chaotic attractor. This remains true for escape over both regular and fractal basin boundaries. It is therefore possible to analyze the stability and control of chaotic systems in much the same way as in the case of escape from a regular attractor. A key problem in all such analyses is determination of the boundary conditions. In many situations they can be found directly through analysis of the geometrical structure of the attractor and/or of the boundary of its basin of attraction, thus avoiding extensive numerical simulation. This applies, in particular, to escape via the primary homoclinic tangenicies closest to the basin boundary, or via homoclinic structure in a fractal boundary.

In the more general case, however, we have to analyze real escape trajectories in order to specify boundary conditions. The prehistory approach is then very powerful. Such analyses demonstrate that, in many cases, escape proceeds via a sequence of jumps between saddle cycles embedded in the chaotic attractor, in the basins of attraction, or in the attractor boundaries. In turn the saddle cycles are closely connected with homoclinic structures (bifurcations). We have found that the escape process depends significantly on the type of chaotic attractor under consideration. Reaching a detailed understanding of the differences in escape from hyperbolic, quasi-hyperbolic, and different kinds of nonhyperbolic chaotic attractors remains a challenge and a direction of future investigation.

\section{References}

Afraimovich, V. S., Bykov, V. V. \& Shilnikov, L. P. [1983], "On structurally unstable attracting limit sets of Lorenz-attractor type," Trans. Moscow Math. Soc. 44, 153-216.

Anishchenko, V. S., Luchinsky, D. G., McClintock, P. V. E., Khovanov, I. A. \& Khovanova, N. A. [2002], "Fluctuational escape from a quasi-hyperbolic attractor in the Lorenz system," JETP 94(4), 821-833.

Anishchenko, V. S., Vadivasova, T. E., Kopeikin, A. S., Kurths, J. \& Strelkova, G. I. [2001], "Effect of noise on the relaxation to an invariant probability measure of nonhyperbolic chaotic attractors," Phys. Rev. Lett. 87(5), 054101.

Beale, P. D. [1989], "Noise-induced escape from attractors in one-dimensional maps," Phys. Rev. A 40(7), 3998-4003. 
Beri, S., Mannella, R., Luchinsky, D. G., Silchenko, A. N. \& McClintock, P. V. E. [2005], "Solution of the boundary value problem for optimal escape in continuous stochastic systems and maps," Phys. Rev. E 72(3), 036131.

Billings, L., Schwartz, I. B., Morgan, D. S., Bollt, E. M., Meucci, R. \& Allaria, E. [2004], "Stochastic bifurcation in a driven laser system: Experiment and theory," Phys. Rev. E 70(2), 026220.

Bishop, S. R. \& Xu, D. [1996], "Control of chaos innoisy flows," Phys.Rev. E 54(4), 3204-3210.

Cohen, J. K. \& Lewis, R. M. [1967], "A ray method for the asymptotic solution of the diffusion equation," J. Inst. Math. Appl. 3, 266-290.

Dykman, M. I. \& Krivoglaz, M. A. [1979], "Theory of the fluctuational transitions between the stable states of a nonlinear oscillator," Sov. Phys. - JETP 50, 30-37.

Dykman, M. I., Luchinsky, D. G., McClintock, P. V. E. \& Smelyanskiy, V. N. [1996], "Corrals and critical behavior of the distribution of fluctuational paths," Phys. Rev. Lett. 77(26), 5229-5232.

Dykman, M. I., McClintock, P. V. E., Smelyanskiy, V. N., Stein, N. D. \& Stocks, N. G. [1992], "Optimal paths and the prehistory problem for large fluctuations in noise driven systems," Phys. Rev. Let. 68(18), 2718-2721.

Faccioli, P., Sega, M., Pedireva, F. \& Orland, H. [2006], "Dominant pathways in protein folding," Phys. Rev. Lett. 97(10), 108101.

Freidlin, M. I. \& Wentzel, A. D., Random Perturbations in Dynamical Systems (Springer, New-York, 1984).

Gonchenko, S. V., Shilnikov, L. P. \& Turaev, D. V. [1997], "Quasiattractors and homoclinic tangencies," Computers Math. Applic. 34(2-4), 195-227.

Graham, R., Hamm, A. \& T'el, T. [1991], "Nonequilibrium potentials for dynamical systems with fractal attractors or repellers," Phys. Rev. Lett. 66(24), 3089-3092.

Graham, R. \& Tél, T. [1984], "Existence of a potential for dissipative dynamical systems," Phys. Rev. Lett. 52(1), 9-12.

Grassberger, P. [1989], "Noise-induced escape from attractors," J. Phys. A: Math. Gen. 22(16), 3283-3290.

Grebogi, C., Ott, E. \& Yorke, J. A. [1987], "Basin boundary metamorphoses - changes in accessible boundary orbits," Physica D 24(1-3), 243-262.

Haken, H. [1975], "Analogy between higher instabilities in fluids and lasers," Phys. Lett. A 53(1), 77-78.

Jaeger, L. \& Kantz, H. [1997], "Homoclinic tangencies and non-normal Jacobians - Effects of noise in nonhyperbolic chaotic systems," Physica D 105(1-3), 79-96. 
Jauslin, H. R. [1987], "Nondifferentiable potentials for nonequilibrium steady states," Physica A 144(1), 179-191.

Jung, P. \& Hänggi, P. [1990], "Invariant measure of a driven nonlinear oscillator with external noise," Phys. Rev. Lett. 65(27), 3365-3368.

Khovanov, I. A., Khovanova, N. A., Grigorieva, E. V., Luchinsky, D. G. \& McClintock, P. V. E. [2006], "Dynamical control: Comparison of map and continuous flow approaches," Phys. Rev. Lett. 96(8), 083903.

Khovanov, I. A., Khovanova, N. A. \& McClintock, P. V. E. [2003], "Noise-induced failures of chaos stabilization: Large fluctuations and their control," Phys. Rev. E 67(5), 051102 .

Khovanov, I. A., Luchinsky, D. G., McClintock, P. V. E. \& Mannella, R. [2000], "Fluctuations and the energy-optimal control of chaos," Phys. Rev. Lett. 85(10), 2100-2103.

Kifer, Y. [1990], "A discrete-time version of the Wentzel-Freidlin theory," Ann. Probab. 18(4), 1676-1692.

Kraut, S. \& Feudel, U. [2003], "Enhancement of noise-induced escape through the existence of a chaotic saddle," Phys. Rev. E 67(1), 015204.

Kraut, S. \& Grebogi, C. [2004], "Escaping from nonhyperbolic chaotic attractors," Phys. Rev. Lett. 92(23), 234101.

Lorenz, E. N. [1963], "Deterministic non-periodic flow," J. Atmos. Sci. 20(2), 130-141.

Luchinsky, D. G. [1997], "On the nature of large fluctuations in equilibrium systems: observation of an optimal force," J. Phys. A: Math. Gen. 30(16), L577-L583.

Luchinsky, D. G. [2002], "Deterministic patterns of noise and the control of chaos," Contemporary Phys. 43(5), 379-395.

Luchinsky, D. G., Greenall, M. J. \& McClintock, P. V. E. [2000], "Resonant rectification of fluctuations in a Brownian ratchet," Phys. Lett. A 273(5-6), 316-321.

Luchinsky, D. G. \& Khovanov, I. A. [1999], "Fluctuation-induced escape from the basin of attraction of a quasiattractor," JETP Lett. 69(11), 825-830.

Luchinsky, D. G., Maier, R. S., Mannella, R., McClintock, P. V. E. \& Stein, D. L. [1997], "Experiments on critical phenomena in a noisy exit problem," Phys. Rev. Lett. 79(17), 3109-3112.

Luchinsky, D. G., McClintock, P. V. E. \& Dykman, M. I. [1998], "Analogue studies of nonlinear systems," Rep. Prog. Phys. 61(8), 889-997.

Maier, R. S. \& Stein, D. L. [1997], "Limiting exit location distributions in the stochastic exit problem," SIAM J. Appl. Math. 57(3), 752-790.

McDonald, S. W., Grebogi, C., Ott, E. \& Yorke, J. A. [1985], "Fractal basin boundaries," Physica D 17(2), 125-153. 
Reimann, P., Müller, R. \& Talkner, P. [1994], "Decay of a metastable states with discrete dynamics," Phys. Rev. E 49(5), 3670-3682.

Reimann, P. \& Talkner, P. [1995], "Escape rates for noisy maps," Phys. Rev. E 51(5), 4105-4113.

Schmelcher, P. \& Diakonos, F. K. [1997], "Detecting unstable periodic orbits of chaotic dynamical systems," Phys. Rev. Lett. 78(25), 4733-4736.

Schroer, C. G., Ott, E. \& Yorke, J. A. [1998], "Effect of noise on nonhyperbolic chaotic attractors," Phys. Rev. Lett. 81(7), 1397-1400.

Serban, R., Koon, W. S., Lo, M. W., Marsden, J. E., Petzold, L. R., Ross, S. D. \& Wilson, R. S. [2002], "Halo orbit mission correction manoeuvers using optimal control," Automatica 38(4), 571-583.

Silchenko, A. N., Beri, S., Luchinsky, D. G. \& McClintock, P. V. E. [2003a], "Fluctuational transitions through a fractal basin boundary," Phys. Rev. Lett. 91(17), 174104.

Silchenko, A. N., Beri, S., Luchinsky, D. G. \& McClintock, P. V. E. [2005], "Fluctuational transitions across different kinds of fractal basin boundaries," Phys. Rev. E 71(4), 046203.

Silchenko, A. N., Luchinsky, D. G. \& McClintock, P. V. E. [2003b], "Noise-induced escape through a fractal basin boundary," Physica A 327(3-4), 371-377.

Smale, S. [1966], "Structurally stable systems are not dense," Am. J. Math. 88(2), 491.

Smelyanskiy, V. N., Luchinsky, D. G., Stefanovska, A. \& McClintock, P. V. E. [2005], "Inference of a nonlinear stochastic model of the cardiorespiratory interaction," Phys. Rev. Lett. 94(9), 098101.

Sole, R. V., Gamarra, J. G. P., Ginovart, M. \& Lopez, D. [1999], "Controlling chaos in ecology: From deterministic to individual-based models," Bull. Math. Biol. 61(6), $1187-1207$.

Soskin, S. M. [1999], "Large fluctuations in multiattractor systems and the generalized Kramers problem," J. Stat. Phys. 97(3-4), 609-676.

Soskin, S. M., Luchinsky, D. G., Mannella, R., Neiman, A. B. \& McClintock, P. V. E. [1997], "Zero-dispersion nonlinear resonance," Int. J. of Bifurcation and Chaos 7(4), 923-936.

Ventcel, A. D. \& Freidlin, M. I. [1970], "On small random perturbations of dynamical systems," Uspehi. Mat. Nauk. 25, 1-56.

Weiss, C. O., Abraham, N. B. \& Hubner, U. [1988], "Homoclinic and heteroclinic chaos in a single-mode laser," Phys. Rev. Lett. 61(14), 1587-1590.

Weiss, C. O., Hubner, U., Abraham, N. B. \& Tang, D. Y. [1995], "Lorenz-like chaos in $\mathrm{NH}_{3}$-FIR lasers," Infrared. Phys. Technol. 36(1), 489-512. 
Zhou, C. S., Kurths, J., Allaria, E., Boccaletti, S., Meucci, R. \& Arecchi, F. T. [2003], "Constructive effects of noise in homoclinic chaotic systems," Phys. Rev. E 67(6), 066220. 